\title{
Selectively Modified Porous Silicon Nanoparticles as Sonosensitizers of Therapeutic Ultrasound
}

\author{
Andrey Sviridov ${ }^{1}$, Konstantin Tamarov ${ }^{1,2}$, Victor Timoshenko ${ }^{1,3}$, Valery Andreev ${ }^{1}$ \\ ${ }^{1}$ Lomonosov Moscow State University, Department of Physics \\ 119991 Moscow, Russia \\ ${ }^{2}$ University of Eastern Finland, Department of Applied Physics \\ 70211, Kuopio, Finland \\ ${ }^{3}$ National Research Nuclear University “MEPhI” (Moscow Engineering Physics Institute), International Laboratory \\ "BioNanophotonics" \\ 115409 Moscow, Russia \\ asagittarius89@gmail.com; k.tamarov@gmail.com; victor_timoshenk@mail.ru; andreev@acs366.phys.msu.ru
}

\section{Extended Abstract}

One of the most actively developed methods for the therapy of some types of cancer is HIFU-surgery using high intensity focused ultrasound [1]. For its utilization, HIFU therapy involves extremely high acoustic intensities $(\sim 0.1-1$ $\mathrm{kW} / \mathrm{cm}^{2}$ ), which may cause too negative influence on healthy tissues of a human body. HIFU surgery is limited by the USI selectivity, which is necessary to achieve therapeutic effect relative to vast tumors and individual cells. The effectiveness of ultrasound (USI) for biomedical purposes can be significantly improved by several substances enhancing its action, or sonosensitizers [2]. Sonosensitizers based on porous silicon (PSi), which properties of biocompatibility [3] and biodegradability [4] have been proved in various in vitro and in vivo experiments, can be used as such agents. The purpose of the present work is experimental and theoretical study of thermal and cavitation effects in aqueous suspensions of mesoporous silicon nanoparticles (PSiNPs).

The PSi films were formed by a standard method of electrochemical etching of crystalline silicon wafers. The samples of selectively modified PSiNPs with hydrophilic outer surface and hydrophobic pore walls were fabricated using hexane as a nano-stopper of pore oxidation and high-energy grinding of PSi films in aqueous medium. TEM, DLS and IRspectroscopy techniques were used to determine size distributions and surface chemistry of the prepared samples.

The scheme of experimental setup used for USI measurements was as followed. A hollow cuvette filled with an investigated sample was placed into a water tank and exposed to the field of calibrated $\mathrm{MHz}$ transducer. Temperature was controlled by E-type thermocouples. The transmitted signal was detected with a sensitive hydrophone. The experiment was automated via a program developed in the LabVIEW environment, which provided control of the generated signal and measurement of time, signal spectrum detected by the hydrophone and temperature.

The spectrum of transmitted signal registered by the hydrophone was obtained using FFT. Besides the fundamental harmonic, it contained multiple harmonics including the subharmonic, which is an evidence of active cavitation in the sample. Time dependences of the main harmonic amplitudes (the half, fundamental and second ones) for the samples of PSiNP suspensions and pure water at various USI intensities were recorded.

Average magnitudes of subharmonic for the samples of oxidized, selectively modified PSiNPs and pure water under exposure to ultrasound of different intensities were obtained. It was revealed that the intensity threshold for the suspension of PSiNPs was significantly lower as compared to water, while the lowest values were observed for the sample of selectively modified SiNPs. This effect can be explained by the presence of nucleation bubbles in the porous structure of nanoparticles, which promote the cavitation process [5].

The heating of PSiNP suspensions and pure water was measured as well. The heating of suspensions was much stronger because of the additional absorption of ultrasonic waves by the nanoparticles [6].

The results of the above experiments were numerically verified using Gilmore-Akulichev model of cavitation bubble dynamics [7] and heat-transfer equation in viscous medium with solid inclusions [8]. 
The obtained experimental results demonstrate outstanding sonosensitizing properties of selectively modified PSiNPs for the lowering of cavitation thresholds and initiation of USI-induced hyperthermia in aqueous media under exposure to the therapeutic USI. These remarkable properties of biodegradable and low-toxic PSiNPs can find applications in the sonodynamic therapy of cancer.

Authors acknowledge the financial support of this work by the Russian Scientific Foundation (grant № 16-1310145).

\section{References}

[1] C. R. Hill, J. C. Bamber and G. R. ter Haar, Physical Principles of Medical Ultrasonics. Second Edition, John Wiley \& Sons, Ltd, 2004.

[2] J. C. Rewcastle, "High intensity focused ultrasound for prostate cancer: a review of the scientific foundation, technology and clinical outcomes," TCRT, vol. 5, no. 6, pp. 619-625, 2006.

[3] L. A. Osminkina, M. B. Gongalsky, A. V. Motuzuk, V. Yu. Timoshenko and A. A. Kudryavtsev, "Silicon nanocrystals as photo- and sono-sensitizers for biomedical applications," Appl. Phys. B: Laser Opt., vol. 105, no. 3, pp. 665-668, 2011.

[4] S. P. Low, N. H. Voelcker, L. T. Canham and K. A. Williams, "The biocompatibility of porous silicon in tissues of the eye," Biomaterials, vol. 30, no. 15, p. 2873-2880, 2009.

[5] A. P. Sviridov, L. A. Osminkina, A. L. Nikolaev, A. A. Kudryavtsev, A. N. Vasiliev and V. Yu. Timoshenko, "Lowering of the cavitation threshold in aqueous suspensions of porous silicon nanoparticles for sonodynamic therapy applications," Appl. Phys. Lett., vol. 107, no. 123107, 2015.

[6] A. P. Sviridov, V. G. Andreev, E. M. Ivanova, L. A. Osminkina, K. P. Tamarov and V. Yu. Timoshenko, "Porous silicon nanoparticles as sensitizers for ultrasonic hyperthermia," Appl. Phys. Lett., vol. 103, no. 193110, 2013.

[7] W. Kreider, L. A. Crum, M. R. Bailey and O. A. Sapozhnikov, "A reduced-order, single-bubble cavitation model with applications to therapeutic ultrasound," J. Acoust. Soc. Am., vol. 130, no. 5, pp. 3511-3530, 2015.

[8] R. J. Urick, "The absorption of sound in suspensions of irregular particles," J. Am. Stat. Assoc., vol. 20, no. 3, 1948. 